\title{
Exact Nonnull Wavelike Solutions to Gravity with Quadratic Lagrangians.
}

\author{
Mark D. Roberts, \\ Department of Mathematics and Applied Mathematics, \\ University of Cape Town, \\ Rondbosch 7701, \\ South Africa \\ roberts@gmunu.mth.uct.ac.za \\ June 20, 2021
}

Published: Int.J.Mod.Phys. 9(1994)167-179.

Eprint: gr-qc/9904007

Comments: 16 pages, no diagrams or tables, LaTex2e.

3 KEYWORDS:

Graviational Waves: Quadratic Lagrangians: Exact Solutions.

1999 PACS Classification Scheme:

http://publish.aps.org/eprint/gateway/pacslist

$04.30+\mathrm{x}, 04.20 \mathrm{Jb}$

1991 Mathematics Subject Classification:

http://www.ams.org/ms

83C $35,83 \mathrm{C} 15$ 


\begin{abstract}
Solutions to gravity with quadratic Lagrangians are found for the simple case where the only nonconstant metric component is the lapse $N$ and the Riemann tensor takes the form $R_{. i t j}^{t}=-k_{i} k_{j}, i, j=1,2,3$; thus these solutions depend on cross terms in the Riemann tensor and therefore complement the linearized theory where it is the derivatives of the Riemann tensor that matter. The relationship of this metric to the null gravitational radiation metric of Peres is given. Gravitaional energy Poynting vectors are construcetd for the solutions and one of these, based on the Lanczos tensor, supports the indication in the linearized theory that nonnull gravitational radiation can occur.
\end{abstract}

\title{
1 Introduction
}

In general relativity it is thought that the assumption of the field equations is sufficient to ensure that gravitational radiation is null (i.e. travels at the speed of light). It is not necessarily the case that gravitational radiation is null [1], but in general relativity four facts support this view. The first is that in the linear approximation, with the harmonic gauge, the field equations reduce to $\square h_{a b}=0$, which has only null wave solutions. The weak field linear approximation may no do justice to the non;inearity of the fielf equations; for example, on a de Sitter background, with cosmological constant $\Lambda$, perturbations obey the Fierz-Pauli equation $\left(\square-m^{2}\right) h_{a b}=0$, with mass $m^{2}=2 \Lambda / 3$ 円. The second fact is that exact solutions of Einstein's field equations which are good models of gravitational radiation represent null radiation. The third is that the charateristics (or shock wave soltuions) of the field equations are null; however, this is not a particularly compelling reason - for example, the characteristics of the massive Klein-gordon equation are null [2]. The fourth is that as a consequence of choosing a metric geometry (where $\nabla_{c} G_{a b}=0$ ), there is the equation $\square g_{a b}=0$, which appears to be massless; this has led to the construction of nonmetric massive theories of gravity obeying the equation $(\square+m) g_{a b}=0$ [3].

One of the most important variants of general relativity is the theory with quadratic terms added to the Lagrangian. Einstein's field equations of general relativity can be derived from Hilbert's Lagrangian [4]. This Lagrangian was generalized to include terms of higher order in the early days of relativity, by Pauli [5], Bach [6] and others. Recently [7] [8], the relevance of quadratic Lagrangian theories to the shape of galaxies has been discused.

\footnotetext{
${ }^{1}$ footnote added 1999, this calculation is done section 6 gr-qc/9812091
} 
When quadratic Lagrangian theories are linearized [9] [10], it is found that the field equations reduce to two wave equations with masses fixed by the values of the coupling constants. In one case the massis positive, leading to slower-than-light waves, and in the other it is negative, leading ot fasterthan-light waves. The initial value formulation of quadratic Lagrangian theory [11], suggests that these masses are present in the full nonlinear theory. Here the existence of exact nonnull wavelike solutions to the quadratic Lagrangian theory is investigated. The method used is to start with a modification of the Peres' wave [12], where the modification entails changing from Peres' dependence on a null coordinate to a nonnull coordinate. Using of coordinate transformations, when the coordinate is timelike the metric is shown to be equivalent to a metric with the lapse $N$ as the only varying component of the metric, and when the metric is spacelike the metric is shown to be static. The Peres metric itself has been discussed in the context of quadratic Lagrangian theories by Buchdahl [13] and Madsen [14]; the metric they use has cylindrical symmetry and depends on a null coordinat, whereas the solutions here do not have these properties. The conventions used are those of Hawking and Ellis [15].

\section{Quadratic Lagrangians}

The action is taken to be of the form

$$
S=\int g^{\frac{1}{2}}\left[\mathcal{L}_{m}+\kappa^{-2}(R-2 \Lambda)+b C^{2}+p R^{2}\right] d x^{4},
$$

where $\mathcal{L}_{m}$ is the matter Lagrangian, the $R$ term is the Hilbert [4] action, $\Lambda$ is the cosmological constant, the $C^{2}$ term is the Bach [6] action with $C^{2}=C_{a b c d} C^{a b c d}$ being the square of the Weyl tensor, and the $R^{2}$ term is the Pauli [5] action. Such nomenclature is historically simplistic; for example, Pauli was considering theories with nonmetric connection. Linearization [9] [10] shows that the coupling constants can be identified with the masses

$$
\kappa^{-2}=\frac{c m_{P L}^{2}}{16 \pi \hbar}, \quad m_{P L}^{2}=\frac{c \hbar}{G}, \quad b=\frac{-1}{2 \kappa^{2} m_{R B}^{2}}, \quad p=\frac{+1}{6 \kappa^{2} m_{W P}^{2}} .
$$

For $\Lambda=0$, there are eight physical degrees of freedom, two for the massless graviton corresponding to the Hilber term, one massive scalar corresponding to the Pauli term, and five massive spin 2 poltergeists corresponding to the Bach term. The sign of these terms can also be considered from the point of view of cosmological stability [16]. 
Varying 2.1 gives the field equations

$$
\kappa^{-2}\left(G_{a b}+\Lambda g_{a b}\right)+M_{a b}+X_{a b}=T_{a b},
$$

where

$$
\begin{aligned}
G_{a b}= & R_{a b}-\frac{1}{2} g_{a b} R, \\
M_{a b}= & -2 b \square R_{a b}+2\left(p+\frac{b}{3}\right) R_{; a b}-\left(2 p-\frac{b}{3}\right) g_{a b} \square R, \\
X_{a b}= & 4 b R_{a c d b} R_{. .}^{c d}-2\left(p-\frac{2 b}{3}\right) R R_{a b} \\
& +g_{a b}\left[b R_{c d} R_{. .}^{c d}+\frac{1}{2}\left(p-\frac{2 b}{3}\right) R^{2}\right] .
\end{aligned}
$$

The trace of 2.3 is

$$
\kappa^{-2}(4 \Lambda-R)-6 p \square R=T,
$$

with $T=T_{c}{ }^{c}$, which leads to the alternative form of the field equations

$$
\kappa^{-2}\left(R_{a b}-g_{a b} \Lambda\right)+X_{a b}+Z_{a b}=S_{a b},
$$

where

$$
\begin{aligned}
& Z_{a b}=-2 b \square R_{a b}+\left(p+\frac{b}{3}\right)\left[2 R_{; a b}+g_{a b} \square R\right], \\
& S_{a b}=T_{a b}-\frac{1}{2} g_{a b} T,
\end{aligned}
$$

and it is this form of the field equations that is used here. The stress tensor is taken to vanish or to be that of a perfect fluid,

$$
S_{a b}=(\mu+\nu) U_{a} U_{b}+\frac{1}{2}(\mu-\nu) g_{a b}
$$

where $\mu$ and $\nu$ are respectively the density and the pressure of the fluid. $U_{a}$ is a unit timelike vector field,

$$
U_{a} U^{a}=-1,
$$

with acceleration, expansion and projection,

$$
\begin{aligned}
A^{a} & =U_{; b}^{a} u^{b}=\dot{U}^{a}, \\
\Theta & =u_{; a}^{a}, \\
i^{a b} & =g^{a b}+U^{a} U^{b},
\end{aligned}
$$


respectively. The first and second conservation equations are

$$
\begin{aligned}
& \mu_{a} U^{a}+(\mu+\nu) \Theta=0, \\
& (\mu+\nu) A^{a}+i^{a b} \nu_{b}=0,
\end{aligned}
$$

respectively.

\section{Poynting Vectors}

In a nonvacuum space-time the speed of a gravitational wave can be measured relative to the matter present. For example, in a cosmological model it can be compared to the speed of the comoving fluid velocity vector; on a galactic scale the speed of a gravitational wave emitted from the center of the galaxy could be estimated be comparing it to the speed of co-occurring electromagnetic radiation, or possibly by direct methods. The metrics that will be here have the property 6.1, where the first conservation implies that the density of a fluid is at rest in this coordinate system, so that there is a possiblity of measuring the speed of a gravitational wave with respect to this fluid. So far the field equations coupled to a perfect fluid have proved intractable, and only solutions with zero stress have been found; thus for a timelike wave it is possible to move to a coordinate system where the wave appears to be at rest, and as there is no matter present this criterion cannot be used to measure wave speed. Another criterion is needed and the one suggested here is to construct Poynting vectors representing the speed of energy transfer: whether the Poynting vector is timelike, null or spacelike will be taken to support the belief that the gravitational wave is timelike, null or spacelike. If the principle of equivalence holds, then the energy of the gravitaional field appears to be nonexistent to an observer in free fall, and consequently a Poynting vector for gravitaional energy gives at best only an indication of the real energetics involved. To sum up: whether a gravitational enery Poynting vector is timelike, null or spacelike can be regarded as measuring whether the gravitational wave is timelike, null or spacelike; alternatively it can be considered as a measure of the appropriatenees, or otherwise, of the Poynting vector.

There are tensors constructed out of products and derivatives of the Riemann tensor, which can represent the square of gravitational energy [17] There are a large number of possible combinations [18]. An often-used com- 
bination is produced by first Matté-decomposing the Weyl tensor

$$
\begin{aligned}
C_{a b c d}=R_{a b c d} & +\frac{1}{6} R\left(g_{a c} g_{d b}-g_{a d} g_{c b}\right) \\
& +\frac{1}{2}\left(g_{a d} R_{c b}-g_{a c} R_{d b}+g_{b c} R_{d a}-g_{d b} R_{c a}\right)
\end{aligned}
$$

into its electric and magnetic parts,

$$
E_{a b} \equiv C_{a m b n} U^{m} U^{n}, \quad B_{a b} \equiv * C_{a m b n} U^{m} U^{n}
$$

where $U_{a}$ is a timelike vector field and $* C_{a b c d}=\frac{1}{2} \sqrt{-g} \epsilon_{a b c d} C_{. . c d}^{m n}$, and then defining

$$
T_{a b c d} \equiv C_{a m b n} C_{c . d .}^{m}+* C_{a m b n} * C_{c . d .}^{m}{ }^{n},
$$

as an energy squared tensor, to give the Poynting vector

$$
\begin{aligned}
P^{a} & \equiv T_{. b c d}^{a} U^{b} U^{c} U^{d} \\
& =C_{. m b n}^{a} E_{. .}^{m n} U^{b}+* C_{. m b n}^{a} B_{. .}^{m n} U^{b} .
\end{aligned}
$$

Energy tensors of the correct dimension can be constructed using the Lanczos tensor [19]. Again there are a large number of possibilities, with the added complication of the choice of gauges possible for the Lanczos tensor. Here it is assumed that both the Lanczos algebraic

$$
3 \chi_{a} \equiv H_{a . b}^{b}=0
$$

gauge and differential

$$
D_{a b} \equiv H_{a b . ; c}^{c},
$$

gauge ave been applied. Subject to these gauges the Weyl tensor is recovered as a linear diferential equation in the Lanczos tensor

$$
\begin{aligned}
C_{a b c d} & =H_{a b c ; d}-H_{a b d ; c}+H_{c d a ; b}-H_{c d b ; a} \\
& -g_{a c} H_{b . d ; e}^{e}+g_{a d} H_{b . d c ; e}^{e}-g_{b d} H_{a . c ; e}^{e}+g_{b c} H_{a . d ; e}^{e}
\end{aligned}
$$

The Lanczos tensor has the symmetries

$$
\begin{aligned}
& H_{a b c}=-H_{b a c}, \\
& H_{a b c}=H_{c b a}-H_{c a b} .
\end{aligned}
$$


Two of the more successfu; energy tensors are

$$
\begin{aligned}
L_{a b} & =H_{a . .}^{c d} H_{b c d}+* H_{a . .}^{c d} * H_{b c d} \\
& =2 H_{a . .}^{c d} H_{b c d}-\frac{1}{2} g_{a b} H_{c d e} H_{\ldots}^{c d e}, \\
M_{a b} & =2 H_{a . b ; c}^{c} .
\end{aligned}
$$

Their Poynting vectors can be defined as

$$
\begin{aligned}
O^{a} & \equiv L_{. .}^{a b} U_{b}, \\
Q^{a} & \equiv M_{. .}^{a b} U_{b},
\end{aligned}
$$

where $U_{a}$ is a timelike vector.

\section{The Metric}

The line element is taken to be of the form

$$
d s^{2}=-d t^{2}+d x^{2}+d y^{2}+d z^{2}+h(u, x, y) d u^{2},
$$

where

$$
u \equiv l t+z .
$$

For $l=1, u$ is null and this is Peres' metric; for $l^{2}<1$ or $l^{2}>1, u$ is spacelike or timelike respectively. For $l^{2}<1$ define

$$
\sqrt{1-l^{2}} \cdot z^{\prime} \equiv l t+z \equiv u, \quad \sqrt{1-l^{2}} \cdot t^{\prime} \equiv t+l z .
$$

Then the metric becomes

$$
d s^{2}=-d t^{2}+d x^{2}+d y^{2}+\left[1+\left(1-l^{2}\right) h\left(z^{\prime}, x, y\right)\right] d z^{\prime 2},
$$

which is manifestly static. For $l^{2}>1$ define

$$
\sqrt{l^{2}-1} \cdot t^{\prime \prime} \equiv l t+z \equiv u, \quad \sqrt{l^{2}-1} \cdot z^{\prime \prime} \equiv t+l z .
$$

Then the metric becomes

$$
d s^{2}=-\left[1+\left(1-l^{2}\right) h\left(\sqrt{l^{2}-1} \cdot t^{\prime \prime}, x, y\right)\right] d t^{\prime \prime 2}+d x^{2}+d y^{2}+d z^{\prime \prime 2} .
$$


This metric has the lapse $N$ as the only varying component of the metric and may be written in the form

$$
d s^{2}=-N^{2} d t^{2}+d x^{2}+d y^{2}+d z^{2} .
$$

When the lapse is of the variables seperable form

$$
N=H(t) \alpha(x, y),
$$

the time dependence can be absorbed into the metric, showing that the metric is static.

\section{The Field Equations}

The line element 4.7 gives the Christoffel symbols

$$
\left\{\begin{array}{l}
t \\
t t
\end{array}\right\}=\frac{\dot{N}}{N}, \quad\left\{\begin{array}{l}
t \\
t i
\end{array}\right\}=\frac{N_{i}}{N}, \quad\left\{\begin{array}{l}
i \\
t t
\end{array}\right\}=N N^{i},
$$

where $i, j=x, y, z$ and $\dot{N}=\partial-t N$. The Riemann tensor, Ricci tensor and Ricci scalar have nonvanishing components:

$$
\begin{aligned}
R_{. i t j}^{t} & =-\frac{N_{i j}}{N}, \quad R_{. t j t}^{i}=N N_{. j}^{i}, \\
R_{t t} & =N N_{i .}^{i}, \quad R_{i j}=-\frac{N_{i j}}{N}, \\
R & =-2 \frac{N_{i .}{ }^{i}}{N} .
\end{aligned}
$$

The first covariant derivatives of the Riemann tensor are

$$
\begin{aligned}
R_{i t j t ; t} & =N^{2}\left(\frac{N_{i j}}{N}\right)^{\circ}, \quad R_{i t j t ; k}=N^{2}\left(\frac{N_{i j}}{N}\right)_{k}, \\
R_{j t i k ; t} & =N_{i} N_{j k}-N_{k} N_{j i} .
\end{aligned}
$$

The first covariant derivatives of the Ricci tensor can be found by contraction; note in particular

$$
R_{i t ; t}=N_{.}^{j} N_{i j}-N_{i} N_{j .}{ }^{j} .
$$


The second covariant derivatives of the Riemann tensor are

$$
\begin{aligned}
R_{i t j t ; t t}= & N^{3}\left\{\left[\frac{1}{N}\left(\frac{N_{i j}}{N}\right)^{\circ}\right]^{\circ}-\left[N\left(\frac{N_{i j}}{N}\right)_{k}\right]^{k}\right\} \\
& +N^{4}\left(\frac{N_{i j}}{N}\right)_{k}^{k}-N N^{k}\left(R_{j t i k ; t}+R_{i t j k ; t}\right), \\
R_{j t i k ; t t}= & N^{2}\left[\frac{1}{N^{2}}\left(n_{i} N_{j k}-N_{k} N_{j i}\right]^{\circ}\right. \\
& -N\left[N_{i}\left(\frac{N_{j k}}{N}\right)^{\circ}+N_{k}\left(\frac{N_{j k}}{N}\right)^{\circ}\right] \\
R_{i j k l ; t t}= & \frac{2}{N}\left(N-i N-k N_{j l}-N_{i} N_{l} N_{j k}-N_{j} N_{k} N_{i j}+N_{l} N_{j} N_{i k}\right), \\
R_{i t j t ; k l}= & N^{2}\left(\frac{N_{i j}}{N}\right)_{k l} .
\end{aligned}
$$

The tensors 2.9 and 2.6 are

$$
\begin{aligned}
Z_{t t}= & 2\left(p-\frac{2 b}{3}\right) N\left[N g^{a b}\left(\frac{N_{i .}^{i}}{N}\right)_{a}\right]_{b}-\frac{4 b N_{.}^{i}}{N} R_{i t ; t} \\
& +4\left(p+\frac{b}{3}\right) N N^{i}\left(\frac{N_{j .}^{j}}{N}\right)_{i}, \\
Z_{i t}= & -2 b\left[-N\left(\frac{R_{i t ; t}}{N^{3}}\right)^{\circ}+\frac{1}{N^{2}}\left(N_{i} \dot{N}_{j .}^{j}-N^{j} \dot{N}_{i j}\right)\right] \\
& -4\left(p+\frac{b}{3}\right) N\left[\frac{1}{N}\left(\frac{N_{j .}^{j}}{N}\right)^{\circ}\right]_{i} \\
Z_{i j}= & -2 b\left\{\frac{1}{N}\left[N g^{a b}\left(\frac{N_{i j}}{N}\right)_{a}\right]_{b}-\frac{1}{N^{3}}\left(N_{i} R_{j t ; t}+N_{j} R_{i t ; t}\right)\right\} \\
& +\left(p+\frac{b}{3}\right)\left\{-4\left(\frac{N_{k .}^{k}}{N}\right)_{i j}-\eta_{i j} \frac{2}{N}\left[N g^{a b}\left(\frac{N_{k .}^{k}}{N}\right)_{a}\right]_{b}\right\},
\end{aligned}
$$

and

$$
X_{t t}=3 b N_{i j} N_{. .}^{i j}+\left(2 p-\frac{7 b}{3}\right) N_{i .}^{i} N_{j .}^{j},
$$




$$
\begin{aligned}
X_{i t} & =0 \\
X_{i j} & =-4 p\left(p+\frac{b}{3}\right) \frac{N_{i j} N_{k .}^{k}}{N^{2}}+\frac{\eta_{i j}}{N^{2}}\left(b N_{k l} N_{. .}^{k l}+\left(2 p-\frac{b}{3}\right) n^{l} l . N_{k .}^{k}\right)
\end{aligned}
$$

respectively.

\section{Solutions to the Field Equations}

From vacuum general relativity, comparing the Ricci tensor 4.3 and the Riemann tensor 4.2 we see that the only solution is flat space. For a perfect fluid the first and second conservation equations 2.14 become

$$
\begin{aligned}
\mu^{0} & =0 \\
(\mu+\nu) \frac{N_{i}}{N} & +\nu_{i}=0
\end{aligned}
$$

respectively. The stress is given by

$$
\begin{aligned}
S^{t} t . & =-\frac{1}{2}(3 \mu+\nu), \\
S_{i j} & =\frac{1}{2} \eta_{i j}(\mu-\nu) .
\end{aligned}
$$

For the perfect fluid in general relativity, the $S_{x y}, S_{x z}, S_{z y}$ equations show that $N$ is a function of one spatial coordinate, say $x$, and then comparing $S_{x x}$ and $S_{y y}$ shows that this vanishes; again the only solution is flat space.

In general the firld equations constructed in Sec 5 are too complex to solve, especially because of the occurrence of $R_{i t ; t}$. Taking

$$
x=r \sin \theta, \quad y=r \cos \theta,
$$

gives

$$
\begin{aligned}
& R_{r t ; t}=\frac{1}{r^{2}}\left(N_{\theta} N_{\theta, r}-\frac{N_{\theta}^{2}}{r N}-N_{r} N_{\theta, \theta}+2 r N_{r}^{2}\right)+N_{z} N_{r z}-N_{r} N_{z z} \\
& R_{\theta t ; t}=N_{r} N_{\theta, r}-\frac{N_{r} N_{\theta}}{r N}-N_{\theta} N_{, r r}+N_{z} N_{\theta z}-N_{\theta} N_{z z} \\
& R_{z t ; t}=N_{r} N_{z r}-N_{z} N_{r r}+\frac{1}{r^{2}}\left[N_{\theta} N_{z \theta}-N_{z}\left(N_{\theta \theta}-2 r N_{r}\right)\right]
\end{aligned}
$$


Requiring $N=N(t, r)$ still leaves $R_{r t ; t}$ nonvanishing. $R_{i t ; t}$ can be made to vanish by assuming that

$$
N=N\left(t, k-i x^{i}\right) .
$$

Then if one lets $k^{2}=k_{i} k^{i}$ the field equations become

$$
\begin{aligned}
S_{. t}^{t}= & -\frac{\Lambda}{\kappa^{2}}-\frac{k^{2} N^{\prime \prime}}{\kappa^{2} N}-\frac{2}{N}\left(p-\frac{2 b}{3}\right)\left[N g^{a b}\left(\frac{N^{\prime \prime}}{N}\right)_{a}\right]_{b} \\
& +2 k^{2}\left(p+\frac{b}{3}\right)\left\{\frac{2}{N}\left[\frac{1}{N}\left(\frac{N^{\prime \prime}}{N}\right)^{\circ}\right]^{\circ}-\frac{k^{2}}{N^{2}}\left[N^{\prime \prime 2}+2 N N^{\prime}\left(\frac{N^{\prime \prime}}{N}\right)^{\prime}\right]\right\} \\
S_{t .}{ }^{\prime}= & -4 k^{2} K_{.}{ }^{i}\left(p+\frac{b}{3}\right) N\left[\frac{1}{N}\left(\frac{N^{\prime \prime}}{N}\right)^{\circ}\right]^{\prime}, \\
S_{i .}{ }^{\prime}= & k_{i} k^{j}\left\{-\frac{N^{\prime \prime}}{\kappa^{2} N}+\frac{2 b}{N}\left[N g^{a b}\left(\frac{N^{\prime \prime}}{N}\right)_{a}\right]_{b}-4 k^{2}\left(p+\frac{b}{3}\right)\left[\left(\frac{N^{\prime \prime}}{N}\right)^{\prime \prime}+\frac{N^{\prime \prime 2}}{N^{2}}\right]\right\} \\
& +2\left(p+\frac{b}{3}\right) \delta_{i .}{ }^{j} k^{2}\left\{-\frac{1}{N}\left[N g^{a b}\left(\frac{N^{\prime \prime}}{N}\right)_{a}\right]_{b}+\frac{k^{2} N^{\prime \prime 2}}{N^{2}}\right\}-\frac{\Lambda}{\kappa^{2}} \delta_{i .}{ }^{j} .
\end{aligned}
$$

First consider the case

$$
p+\frac{b}{3}=\frac{1}{6 \kappa^{2}}\left(\frac{1}{m_{W P}^{2}}-\frac{1}{m_{R B}^{2}}\right)=0,
$$

and $\Lambda=0$; then the field equations become

$$
\begin{aligned}
S_{a b} & =-2 b\left(\square-\frac{1}{2 b \kappa^{2}}\right) R_{a b} \\
& =\frac{1}{\kappa^{2} m_{R B}^{2}}\left(\square+m_{R B}^{2}\right) R_{a b} .
\end{aligned}
$$

For $S_{a b}=0$, by the use of 5.3 and 5.8 this equation is

$$
0=\left[\frac{1}{N}\left(\frac{N_{i j}}{N}\right)^{\circ}\right]^{\circ}-\left[N\left(\frac{N_{i j}}{N}\right)_{k}\right]^{k}-m_{R B}^{2} N_{i j},
$$

where $\dot{N}=\partial_{t} N$. This has the static solution

$$
N=A(t) \exp \left(a_{0}+a_{1} z-\frac{1}{4} m_{R B}^{2} z^{2}\right)
$$


where $a_{0}$ and $a_{1}$ are constants. This solution can be rotated around the spatial axis, and also transferred into the form 4.1 .

A nonstatic solution can be found by assuming that the lapse $N$ is a function of $w=k_{a} x^{a}$, where $k_{a}$ is a constant. Symbolically this is

$$
N=N\left(-k_{0} t+k_{i} x^{i}\right)=N\left(k_{a} x^{a}\right)=N(w) .
$$

The Riemann tensor takes the simple form

$$
R_{. i t j}^{t}=-k_{i} k_{j} \frac{N^{\prime \prime}}{N}
$$

If one defines

$$
t \equiv u=\frac{l t^{\prime}+z^{\prime}}{\sqrt{l^{2}-1}}, \quad z \equiv \frac{t^{\prime}+l z^{\prime}}{\sqrt{l^{2}-1}},
$$

the metric can be put in the form 3.1 with

$$
h=1-N^{2}\left[u\left(-k_{0}+\frac{k_{z}}{l}\right)+k_{x} x+k_{y} y+k_{z} \frac{\sqrt{l^{2}-1}}{l} z^{\prime}\right] .
$$

If one assumes that $p=-b / 3$ as in 6.7, the field equations are

$$
\begin{gathered}
S_{t .}{ }^{t}=S_{i .}^{i} \\
\kappa^{2} m_{2}^{2} S_{i j}=k_{i} k_{j}\left\{-m_{2}^{2} \frac{N^{\prime \prime}}{N}-\frac{k_{0}^{2}}{N}\left[\frac{1}{N}\left(\frac{N^{\prime \prime}}{N}\right)^{\prime}\right]^{\prime}+\frac{k^{2}}{N}\left[N\left(\frac{N^{\prime \prime}}{N}\right)^{\prime}\right]^{\prime}\right\} .
\end{gathered}
$$

When $S_{a b}=0$ these equations reduce to the single equation

$$
-\frac{k_{0}^{2}}{N}\left[\frac{1}{N}\left(\frac{N^{\prime \prime}}{N}\right)^{\prime}\right]^{\prime}+\frac{k^{2}}{N}\left[N\left(\frac{N^{\prime \prime}}{N}\right)^{\prime}\right]^{\prime}-m_{2}^{2} \frac{N^{\prime \prime}}{N}=0 .
$$

Integrating once, with the constant of integration $a_{1}$, and multiplying by $N$ gives

$$
\left(k^{2} N^{2}-k_{0}^{2}\right)\left(\frac{N^{\prime \prime}}{N}\right)^{\prime}-m_{2}^{2} N N^{\prime}-a_{1} N=0 .
$$

Setting the constant of integration $a_{1}=0$ and integrating again with the constant of integration $a_{2}$ gives

$$
\frac{N^{\prime \prime}}{N}=a_{2}+\frac{1}{2} m_{2}^{2} k^{-2} \ln \left(k^{2} N^{2}-k_{0}^{2}\right) .
$$


This differential equation remains intractable when $m_{2} \neq 0$, but when $m_{2}=$ 0 it has the simple solution

$$
N=C_{+} \exp \left(k_{a} x^{a}\right)+C_{-} \exp \left(-k_{a} x^{a}\right),
$$

where a factor of $\sqrt{a_{2}}$ has been absorbed into $k_{a}$, and thus $\sqrt{a_{2}} k_{a} \rightarrow k_{a}$; because $a_{2}$ is a constant of integration $k_{a}$ may now be complex. For 6.19 the Riemann tensor takes the simple form

$$
R_{. i t j}^{t}=-k_{i} k_{j}
$$

For $p+\frac{b}{3} \neq 0$, inspection of the $S_{t}{ }^{i}$ term 6.7 and the fluid conservation equations 6.1 suggests that it should be possible to integrate the field equations, but so far this has turned out not to be the case. It is known [20] that a sperically symmetric metric is always a solution of the general relativity field equations if a sufficiently large number of other fields and fluids are present. Adding more terms to the stress in 6.7 has not yet given invertible equations. To find $p+\frac{b}{3} \neq 0$ solutionsthe expresion 6.20, i.e. $R_{. i t j}^{t}=-k_{i} k_{j}=-N_{i j} / N$, is substituted back into the field equations 6.7. This assumption amounts to disregarding higher derivatives of the Rieman tensor and investigating only the effect of cross terms; therefore it complements previous linear analysis. Substituting 6.7 gives the field equations

$$
\begin{aligned}
S_{t .}{ }^{t} & =-\frac{k^{2}}{\kappa^{2}}-\frac{\Lambda}{\kappa^{2}}-2 k^{4}\left(p+\frac{b}{3}\right), \\
S_{t .}{ }^{i} & =0 \\
S_{i .}^{i} & =-\frac{k^{2}}{\kappa^{2}}-\frac{3 \Lambda}{\kappa^{2}}+2 k^{4}\left(p+\frac{b}{3}\right), \\
S_{i .}^{j}(i \neq j) & =k_{i} k^{j}\left[-\frac{1}{\kappa^{2}}-4 k^{2}\left(p+\frac{b}{3}\right)\right] .
\end{aligned}
$$

Taking $S_{a b}=0$ gives the solution

$$
\begin{aligned}
k^{2} & =-2 \Lambda=\frac{-1}{4 \kappa^{2}\left(p+\frac{b}{3}\right)} \\
& =\frac{-3}{2\left(m_{W P}^{-2}-m_{R B}^{-2}\right)} .
\end{aligned}
$$




\section{Poynting Vectors for the Metric}

From Eqs. 3.1 and 4.2 the Weyl tensor is found to be

$$
\begin{aligned}
C_{. i t j}^{t}= & -\frac{N_{i j}}{2 N}+\frac{N_{k .}^{k}}{6 N} \eta_{i j}, \\
C_{j k l}^{i}= & \frac{1}{2 N}\left(-\delta_{l}^{i} N_{j k}+\delta_{k}^{i} N_{j l}-\eta_{j k} N_{l}^{i}+\eta_{j l} N_{k}^{i}\right) \\
& -\frac{N_{m .}^{m}}{3 N}\left(\delta_{k}^{i} \eta_{j l}-\delta_{l}^{i} \eta_{j k}\right) .
\end{aligned}
$$

The electric and magnetic parts become

$$
\begin{aligned}
E_{i j} & =\frac{N_{i j}}{2 N}-\frac{N_{k .}^{k}}{6 N} \eta_{i j}, \\
B_{a b} & =0 .
\end{aligned}
$$

The Poynting vector 3.4 is

$$
P^{a}=\frac{\delta_{t}^{a}}{12 N^{3}}\left(3 N_{i j}^{2}-N_{i i}^{2}\right)
$$

and thus

$$
P_{a} P^{a}=\frac{-1}{12^{2} N^{4}}\left(3 N_{i j}^{2}-n_{i i}^{2}\right)^{2},
$$

is always timelike. This Poynting vector appears to give no indication of energy transfer as $P^{t}$ is the only nonvanishing component.

The Lanczos tensor can be calulated by direct methods similar to those used in Ref. 221]. In the Lanczos algebraic and differential gauges it is found to be

$$
H_{. i t}^{t}=-\frac{N_{i}}{3 N}, \quad H_{i j k}=\frac{1}{6 N}\left(N_{j} \eta_{i k}-N_{i} \eta_{j k}\right) .
$$

The energy tensor 3.10 is

$$
L_{t t}=-\frac{N_{i}^{2}}{18}, \quad L_{i t}=0, \quad L_{i j}=\frac{1}{18 N^{2}}\left(5 N_{i} N_{j}-2 \eta_{i j} N_{k}^{2}\right),
$$

and the associated Poynting vector is

$$
O^{a}=-\frac{N_{i}^{2} \delta_{t}^{a}}{18 N^{2}}
$$


thus

$$
O_{a} O^{a}=-\left(\frac{N_{i}^{2}}{18 N^{2}}\right)^{2}
$$

and again this is always timelike.

The energy tensor 3.10 is

$$
\begin{aligned}
& M_{. t}^{t}=-\frac{2}{3}\left(\frac{N_{.}^{i}}{N}\right)_{i}, \quad M_{. t}^{i}=-\frac{2}{3}\left(\frac{N_{.}^{i}}{N}\right)^{\circ}, \\
& M_{i j}=\frac{2 N_{i} N_{j}}{3 N^{2}}-\frac{N_{i j}}{3 N}+\eta_{i j} \frac{N_{. k}^{k}}{3 N}
\end{aligned}
$$

and the associated Poynting vector is

$$
Q_{.}^{a}=\frac{2}{3 N}\left[\left(\frac{N^{i}}{N}\right)_{i},\left(\frac{N^{i}}{N}\right)^{\circ}\right]
$$

giving

$$
Q_{a} Q^{a}=-\frac{4}{9} g^{a b}\left(\frac{N^{i}}{N}\right)_{a}\left(\frac{N_{i}}{N}\right)_{b} .
$$

For 6.20 with either $C_{+}=0$ or $C_{-}=0, Q^{a}=0$; however, for $C=C_{+}=C_{-}$,

$$
Q_{a} Q^{a}=-\frac{4}{9} k^{2}\left(k^{2}-\frac{k_{0}^{2}}{N^{2}}\right) \operatorname{Sech}^{4}\left(k_{a} x^{a}\right) .
$$

For the solution 6.22, 7.12 reduces to

$$
Q_{a} Q^{a}=\frac{-1}{\left(m_{W P}^{-2}-m^{-2}{ }_{R} B\right)}\left(\frac{1}{m_{W P}^{-2}-m_{R B}^{-2}}+\frac{2 k_{0}^{2}}{3 N^{2}}\right) \cdot \operatorname{Sech}\left(k_{a} x^{a}\right) .
$$

When $m_{R B}=0, Q^{a}$ is always timelike; when $M_{R B} \neq 0$ a variety of behaviour is possible, depending on the values of the constants.

\section{Acknowledgements}

I would like to thank the Leverhulme Trust for financial support. 


\section{References}

[1] M.D.Roberts,Spec.Sci.Tec.10,200(1987).

[2] J.L.Synge, Relativity: The General Theory (North-Holland, 1960),p.226.

[3] M.D.Roberts, Ph.D.thesis (University of London, 1986), Chap.4; and gr-qc/9812091

[4] D.Hilbert,Konigl.Nachr.d.Wiss.Gottingen3,395(1915).

[5] W.Pauli,Phys.Zeit.20,457(1919),Eq,(50).

[6] R.Bach,Math.Zeit.9,110(1921).

[7] H.-J.Schmidt,Europhys.Lett.12,667(1990).

[8] M.D.Roberts,Mon.Not.R.asto.Soc.249,339(1991).

[9] K.S.Stelle, Gen.Relativ. Gravit.9,353(1978).

[10] N.H.Barth and S.M.Christensen,Phys.Rev.D28,1876(1983).

[11] D.R.Noakes, J.Math.Phys.24,1846(1982).

[12] A.Peres,Phys.Rev.Lett.3,571(1959).

[13] H.A.Buchdahl,J.Phys.A16,1441(1983).

[14] M.S.Madsen,Class.Quantum Grav.7,87(1990).

[15] S.W.Hawking and G.F.R.Ellis, The Large Scale Structure of SpaceTime (Cambridge Univ. Press,1973),p.84.

[16] A.A.Coley and R.K.Tavakol, Gen.Relativ.Gravit.24,835(1992).

[17] L.Bel, Cah.Phys.16,59(1962).

[18] C.D.Collinson, Proc.Cambridge Philos.Soc.58,346(1962).

[19] M.D.Roberts, Gen.Relativ.Gravit.20,775(1988).

[20] M.D.Roberts, Gen.Relativ.Gravit.21,907(1989),Eq.(A22).

[21] M.D.Roberts, Il Nuovo Cim.B110,1165(1995).gr-qc/9904006. 